\title{
Temperature dependence of Raman-active phonons and anharmonic interactions in layered hexagonal $\mathrm{BN}$
}

\author{
Ramon Cuscó, ${ }^{1}$ Bernard Gil, ${ }^{2}$ Guillaume Cassabois, ${ }^{2}$ and Luis Artús ${ }^{1}$ \\ ${ }^{1}$ Institut Jaume Almera (ICTJA-CSIC), Consejo Superior de Investigaciones Científicas, 08028 Barcelona, Spain \\ ${ }^{2}$ Laboratoire Charles Coulomb (L2C), UMR 5221 CNRS-Université de Montpellier, F-34095, Montpellier, France
}

(Received 19 July 2016; published 20 October 2016)

\begin{abstract}
We present a Raman scattering study of optical phonons in hexagonal BN for temperatures ranging from 80 to $600 \mathrm{~K}$. The experiments were performed on high-quality, single-crystalline hexagonal BN platelets. The observed temperature dependence of the frequencies and linewidths of both Raman active $E_{2 g}$ optical phonons is analyzed in the framework of anharmonic decay theory, and possible decay channels are discussed in the light of density-functional theory calculations. With increasing temperature, the $E_{2 g}^{\text {high }}$ mode displays strong anharmonic interactions, with a linewidth increase that indicates an important contribution of four-phonon processes and a marked frequency downshift that can be attributed to a substantial effect of the four-phonon scattering processes (quartic anharmonicity). In contrast, the $E_{2 g}^{\text {low }}$ mode displays a very narrow linewidth and weak anharmonic interactions, with a frequency downshift that is primarily accounted for by the thermal expansion of the interlayer spacing.
\end{abstract}

DOI: 10.1103/PhysRevB.94.155435

\section{INTRODUCTION}

Hexagonal boron nitride $(h$-BN) belongs to a family of layered materials that has attracted great research interest after the rapid development of the physics and applications of graphene [1]. $h$-BN and graphite are isoelectronic materials which exhibit a similar hexagonal layered structure with similar lattice parameters [2]. Nevertheless, the different electronegativity of the constituents of $\mathrm{BN}$ contributes to the stabilization of the $A A^{\prime}$ layer stacking [3] and to the electrical insulator character of the material. $h$-BN was found to lase at $215 \mathrm{~nm}$ at room temperature (RT), suggesting a direct band gap of $5.97 \mathrm{eV}$ [4]. However, two-photon spectroscopy experiments have recently shown that $h$-BN has an indirect band gap of $5.955 \mathrm{eV}$ and that emission is structured by phonon-assisted recombination [5,6]. The optical emission properties of $h$-BN make it a promising material for ultraviolet laser applications in optical storage, photocatalysis, ophtalmic surgery, and nanosurgery [4].

$h$-BN exhibits strong in-plane bonds with absence of dangling bonds and a lattice constant similar to that of graphene. This makes it a substrate ideally suited for chemical vapor deposition growth of graphene [7]. Because of their high mechanical strength and flexibility, and their electrical insulating character, two-dimensional layers of $h$-BN are also emerging as essential building blocks for flexible and transparent field-effect transistors based on stacking ultrathin layered materials $[1,8]$. In these layered heterostructures, the atomically flat interfaces arising from van der Waals bonding are free of surface states and yield improved electron mobilities [9]. Quite recently, a $h$-BN/black phosphorous/ $h$-BN $\mathrm{THz}$ photodetector has been demonstrated, showing a remarkably high signal-to-noise ratio and high values of the field-effect hole mobility [10]. Furthermore, the basal plane thermal conductivity of $h$-BN is two orders of magnitude higher than that of $\mathrm{SiO}_{2}$, and therefore the $h$-BN dielectric layer enhances the lateral heat spreading from the conducting active layer thus helping to efficiently dissipate heat [11], which is critical for close packed electronic devices.
Despite the high technological interest of $h$-BN, experimental studies on the lattice dynamics of this material are scarce [12]. Early Raman scattering measurements performed on polycrystalline pyrolytic $h$-BN samples detected only the intralayer $E_{2 g}^{\text {high }}$ mode [13]. The low-frequency interlayer mode $\left(E_{2 g}^{\text {low }}\right)$ was later reported by Kuzuba et al. [14]. Raman scattering measurements on microcrystalline powders revealed shifts and broadenings of the $E_{2 g}^{\text {high }}$ mode that were attributed to size effects [15]. More recently, a comprehensive Raman scattering study under resonant excitation revealed a strong enhancement of the scattering susceptibility and allowed the second-order Raman spectrum of $h$-BN to be obtained [16]. Raman scattering can provide valuable information about phonon lifetimes and the anharmonic decay of phonons into other phonons that conserve energy and momentum [17-19]. Recently, we have reported a Raman scattering study of anharmonic decay processes and phonon lifetime in zinc-blende type cubic GaN [20]. Whereas phonon anharmonicities have been studied in detail by means of Raman scattering experiments in the closely related graphite and graphene structures [21,22], no information exists in the literature about phonon anharmonic decay in $h$-BN. The temperature dependence of the Raman frequency and linewidth of the $E_{2 g}^{\text {high }}$ mode was reported for temperatures above RT for a polycrystalline BN coating, and it was concluded that it could not be explained in terms of a simple model involving a single phonon decay channel [23]. Strong coupling of optical phonons to electrons in graphite yields ultrafast electron relaxation via excitation of a hot phonon population, whose lifetime was estimated to be of 7 ps [24]. The build-up of a hot phonon population may limit the performance of devices at high electric fields and/or high temperatures. There are no data on optical phonon lifetimes in $h$-BN available in the literature.

In this paper, we present a Raman scattering study of the temperature dependence of both intralayer and interlayer Raman-active $E_{2 g}$ modes in the 80 to $600 \mathrm{~K}$ temperature range. The observed shifts and broadenings are analyzed in terms of anharmonic phonon decay, taking into account 
the strongly anisotropic thermal expansion of $h$-BN [25]. The experimental results are interpreted in the framework of Cowley's perturbation theory using density functional theory (DFT) calculations of the phonon dispersion and phonon density of states (PDOS). The most likely decay channels for both modes are identified. The temperature behavior of the $E_{2 g}^{\text {high }}$ linewidth of $h$-BN differs substantially from that of graphite, on account of the different mechanisms contributing to anharmonic decay in these two layered crystals, yet it exhibits a considerable frequency downshift with temperature similar to that reported in graphite [21]. The $E_{2 g}^{\text {high }}$ phonon lifetime estimated from the Raman spectra suggests a faster phonon thermalization than in graphite.

The paper is organized as follows. In Sec. II, the experimental setup and the sample preparation are described. Section III introduces the theoretical tools needed to discuss our results. In Sec. III A, the anisotropic thermal expansion of $h$-BN is discussed. Specific calculations of its effect on the phonon frequencies are presented in Sec. IV B. Section III B provides an overview of the phonon anharmonic decay theory that will be used to analyze our experimental data. The particularities presented by similar layered compounds such as graphite and graphene are also discussed in this framework. In Sec. III C, we describe the ab initio DFT calculations carried out to obtain the phonon dispersion and the PDOS, which are used to identify the most likely decay channels. In Sec. IV A, we present the Raman spectra of $h$-BN recorded at temperatures between 80 and $600 \mathrm{~K}$, and in Sec. IV C, we analyze their temperature dependence within the framework described in Sec. III. Finally, Sec. V summarizes the main findings of this work.

\section{EXPERIMENT}

The experiments were carried out on a bulk, singlecrystalline BN platelet of $1.5 \times 1 \times 0.1 \mathrm{~mm}$ supplied by HQ Graphene. For the low-temperature measurements, the sample was mounted on a copper disk with a central 500- $\mu \mathrm{m}$ diameter hole. The sample was glued with silver lacquer around the hole rim to ensure good thermal contact, while the central part of the transparent sample remained clear so that the background signal arising from the silver lacquer was minimized. Measurements from room temperature (RT) down to $80 \mathrm{~K}$ were carried out in a TBT-Air Liquide liquid nitrogen $\left(\mathrm{LN}_{2}\right)$ cryostat equipped with a platinum resistance sensor and a temperature controller. For the high-temperature measurements, a Linkam THMS600 heating stage was employed. The sample was mounted on an in-house-made sample holder consisting of a thin copper disk with a central pillar and 500- $\mu \mathrm{m}$ diameter hole. Good thermal contact was ensured by using a thin coating of conductive silver silicone grease around the hole and between the copper disk and the THMS600 heating block. The temperature was monitored by a thermocouple attached to the central pillar close to the sample location. The sample space was purged with nitrogen gas.

The Raman scattering measurements were carried out in backscattering configuration from the $c$ face using the 514.5-nm line of an $\mathrm{Ar}^{+}$laser. The scattered light was analyzed using a Jobin-Yvon T64000 triple spectrometer equipped with a $\mathrm{LN}_{2}$-cooled charge-coupled device (CCD) detector. The Raman spectra were obtained in the subtractive configuration of the spectrometer with $100-\mu \mathrm{m}$ slits, which corresponds to a spectral bandwidth of $\sim 2.2 \mathrm{~cm}^{-1}$. The laser power on the sample was kept very low to avoid heating effects. The incident power on the sample was of $8 \mathrm{~mW}$ in the macrocamera setup and $700 \mu \mathrm{W}$ in the micro-Raman measurements.

\section{THEORETICAL FRAMEWORK}

The temperature dependence of the first-order Raman line shapes provides information about the anharmonic processes in crystals. In the quasiharmonic approximation, the temperature-induced change in the crystal volume results in a strained crystal and hence in a phonon frequency shift given by

$$
\Delta_{0}=-\omega_{0} \gamma \int_{0}^{T}\left[\alpha_{c}\left(T^{\prime}\right)+2 \alpha_{a}\left(T^{\prime}\right)\right] d T^{\prime},
$$

where $\gamma$ and $\omega_{0}$ are, respectively, the Grüneisen parameter and the harmonic frequency of the mode considered, and $\alpha_{c}$ and $\alpha_{a}$ are the linear thermal expansion coefficients parallel and perpendicular to the $c$ axis. The anharmonic phonon-phonon interaction introduces higher order terms in the Hamiltonian that couple the harmonic eigenstates and give rise to finite phonon lifetimes. These are reflected in measurable frequency shifts and line broadenings in the Raman spectra. Therefore the analysis of the temperature dependence of the Raman spectra yields valuable information about the anharmonic processes.

\section{A. Anisotropic thermal expansion of $h$-BN}

Layered crystals such as graphite and $h$-BN exhibit strongly anisotropic structural properties owing to the highly different inter and intralayer interactions. Whereas the interlayer interactions are dominated by weak van der Waals dispersion forces, the intralayer interactions are governed by the strong $s p^{2}$ bonding. In the extreme case of a single graphene layer, the lowest transverse acoustic modes (bending modes) display a large negative Grüneisen parameter, i.e., their frequencies increase when the in-plane lattice parameter is increased, because such an increase stiffens the out-of-plane undulating ripples of the sheet associated with the acoustic bending modes (membrane effect) [26]. As a result, graphene shows a large negative thermal expansion coefficient $\left(\alpha_{a} \sim-7 \times 10^{-6} K^{-1}\right.$ at RT) [27]. This effect is mitigated in graphite and $h$-BN because of the stacking, but these layered crystals still display negative values of the in-plane thermal expansion coefficient. In contrast, the weak bonding in the $c$ direction causes the thermal expansion in this direction to be large and positive.

A detailed study of the thermal expansion in $h$-BN was carried out by Paszkowicz et al. [25], who determined the lattice parameters of $h$-BN at temperatures between $10 \mathrm{~K}$ and RT by x-ray diffraction using a syncroton-radiation source. They found the negative in-plane thermal expansion coefficient to steadily increase in absolute value for temperatures between 50 and $160 \mathrm{~K}$ and then stabilize at a RT value of $\alpha_{a}=-2.72 \times$ $10^{-6} K^{-1}$. On the other hand, the thermal expansion coefficient in the $c$ direction increases for temperatures up to $160 \mathrm{~K}$ and then stabilizes to a RT value of $\alpha_{c}=3.8 \times 10^{-5} K^{-1}$. In Sec. III C, we will use the temperature dependence of the 


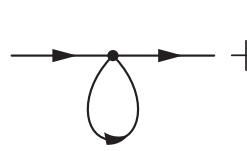

(a) (b)

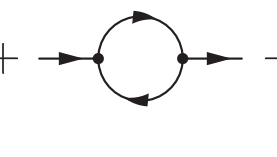

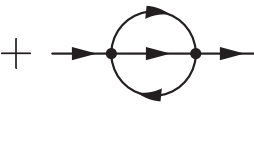

(c)
FIG. 1. Diagrammatic expansion of the phonon self-energy up to second order in perturbation theory. The first-order diagram (a) can be interpreted as a quartic anharmonic correction due to fourphonon scattering (see text), whereas the second-order diagrams (b) and (c) correspond to cubic and quartic anharmonic contributions associated with phonon decay processes into two and three phonons, respectively.

lattice constants derived from these x-ray measurements to evaluate the effect of thermal expansion on the temperaturedependent phonon frequency shifts. For temperatures above RT, we use the lattice parameters derived from the temperature dependence of the thermal expansion coefficient reported in an earlier interferometric study [28].

\section{B. Anharmonic interactions}

The coupling of the harmonic eigenstates by the anharmonic interactions is usually described by perturbation theory [29] through a complex phonon self-energy $\Delta(\omega)+i \Gamma(\omega)$. The imaginary part of the phonon self-energy accounts for the Raman line shape broadening due to the finite phonon lifetime caused by anharmonic phonon decay. This analysis has been successfully applied to a wide number of semiconductors $[17,19,20,30]$. In metallic systems, electron-phonon coupling can also limit the phonon lifetime by creating electron-hole pairs. The electron-phonon coupling can take place in graphite since it is a vanishing gap material, and indeed it has been found to be the dominant effect in the anomalous $E_{2 g}$ linewidth decrease with temperature observed in graphite and graphene [31]. However, because of the wide band gap of $h$-BN, electron-phonon coupling is not relevant in limiting the $E_{2 g}^{\text {high }}$ phonon lifetime of $h$-BN, which, as we shall see in Sec. IV A, exhibits the expected linewidth increase with temperature characteristic of anharmonic decay.

The diagrammatic expansion of the phonon self-energy up to second order in perturbation theory [29] is depicted in Fig. 1. Diagrams (b) and (c) correspond to actual phonon decay processes in which a phonon is annihilated and, respectively, two or three wave-vector and energy conserving phonons are created. These processes limit the phonon lifetime and therefore contribute to the imaginary part of the phonon selfenergy. In contrast, the first-order loop diagram (a) does not involve the annihilation of the phonon and can be interpreted as the wave-vector-conserving elastic scattering of the phonon with phonons in other branches. Such a scattering process does not limit the phonon lifetime and thus it yields a purely real contribution to the phonon self-energy.

To the lowest order in perturbation theory, the imaginary part of the phonon self-energy is given by [29]

$$
\begin{aligned}
\Gamma(\omega)= & \frac{18 \pi}{\hbar^{2}} \sum_{\mathbf{q} j_{1} j_{2}}\left|V_{3}\left(\mathbf{q} j_{1},-\mathbf{q} j_{2}\right)\right|^{2} \times\left[\left(n_{1}+n_{2}+1\right)\right. \\
& \left.\times \delta\left(\omega_{1}+\omega_{2}-\omega\right)+\left(n_{2}-n_{1}\right) \delta\left(\omega_{1}-\omega_{2}-\omega\right)\right],
\end{aligned}
$$

where $V_{3}\left(\mathbf{q} j_{1},-\mathbf{q} j_{2}\right)$ is the third-order coefficient in the expansion of the lattice potential in normal coordinates and $n_{1}$ and $n_{2}$ are the Bose-Einstein occupation factors for the modes $\left(\mathbf{q}, j_{1}\right)$ and $\left(-\mathbf{q}, j_{2}\right)$, respectively. The first term in Eq. (2) corresponds to the decay of a phonon in two lower-frequency phonons whereas the second term describes the creation of a higher energy phonon with the concurrent annihilation of a phonon. Assuming slowly varying Bose-Einstein factors and $V_{3}$ coefficients in the small energy range considered, the imaginary part of the self-energy is proportional to the two-phonon DOS and can be written as [30]

$$
\Gamma(\omega)=\left|V_{3}^{+}\right|^{2}\left(1+n_{1}+n_{2}\right) \rho^{+}(\omega)+\left|V_{3}^{-}\right|^{2}\left(n_{2}-n_{1}\right) \rho^{-}(\omega),
$$

with $\rho^{+}(\omega)$ and $\rho^{-}(\omega)$ the two-phonon sum and difference DOS, and $V_{3}^{+}$and $V_{3}^{-}$the effective third-order anharmonic constants for decay into phonon sums and phonon differences, respectively. Higher-order processes involving decay into three phonons may be necessary to account for line shape broadening as temperature increases [30,32]. If we restrict ourselves to decay processes resulting in a zone center phonon and a pair of opposite wave-vector phonons at zone edge, the contribution to the imaginary part of the phonon self-energy can be approximated by [30]

$$
\begin{aligned}
\Gamma^{(4)}(\omega) \sim & \left|\tilde{V}_{4}^{+}\right|^{2}\left\{\left[n\left(\omega_{1}^{+}\right)+1\right]\left[n\left(\omega_{2}^{+}\right)+1\right]\left[n\left(\omega_{3}^{+}\right)+1\right]\right. \\
& \left.-n\left(\omega_{1}^{+}\right) n\left(\omega_{2}^{+}\right) n\left(\omega_{3}^{+}\right)\right\} \rho^{+}\left(\omega-\omega_{1}^{+}\right) \\
& +\left|\tilde{V}_{4}^{-}\right|^{2}\left\{\left[n\left(\omega_{1}^{-}\right)+1\right]\left[n\left(\omega_{2}^{-}\right)+1\right] n\left(\omega_{3}^{-}\right)\right. \\
& \left.-n\left(\omega_{1}^{-}\right) n\left(\omega_{2}^{-}\right)\left[n\left(\omega_{3}^{-}\right)+1\right]\right\} \rho^{-}\left(\omega-\omega_{1}^{-}\right),
\end{aligned}
$$

where $\omega_{1}$ is the frequency of the zone-center mode considered and $\omega_{2}, \omega_{3}$ are the frequencies of opposite wave-vector phonon modes satisfying energy conservation. In Eq. (4), the first term accounts for the decay into three lower-energy phonons whereas the second term describes an energy-conserving process that entails the annihilation of one phonon.

Since real and imaginary parts of the phonon self-energy are related by a Kramers-Kronig transformation, the corresponding real part of the self-energy, $\Delta(\omega)$, can be evaluated as

$$
\Delta(\omega)=-\frac{2}{\pi} \mathcal{P} \int_{0}^{\infty} \frac{\omega^{\prime}}{\omega^{\prime 2}-\omega^{2}} \Gamma\left(\omega^{\prime}\right) d \omega^{\prime} .
$$

The first-order loop [diagram (a) in Fig. 1] yields an additional purely real contribution to the phonon self-energy that is given by [29]

$$
\Delta^{4 \mathrm{ps}}=\frac{12}{\hbar} \sum_{\boldsymbol{q} j} V^{(4)}\left(\mathbf{0} j_{0}, \mathbf{0} j_{0}, \boldsymbol{q} j,-\boldsymbol{q} j\right)[2 n(\boldsymbol{q} j)+1] .
$$

Here, 4ps stands for four-phonon scattering. Indeed, this is a fourth-order term in the anharmonic potential which can be viewed as a scattering process between the zone center phonon under consideration $\left(\mathbf{0} j_{0}\right)$ and a pair of opposite wave-vector phonons $(-\boldsymbol{q} j)$ and $(\boldsymbol{q} j)$.

Once the phonon self-energy is determined, the Raman line shape can be evaluated as

$$
I(\omega) \propto \frac{\Gamma(\omega)}{\left[\omega_{0}+\Delta_{0}+\Delta(\omega)-\omega\right]^{2}+\Gamma(\omega)^{2}} .
$$


Here, $\Delta_{0}$ is the frequency shift associated with the thermal expansion of the lattice. Note that in the case of a frequency independent self-energy, the phonon line shape is Lorentzian with a full width at half maximum (FWHM) equal to two times the imaginary part of the self-energy. If the frequency dependence of the self-energy is not negligible, it may introduce corrections to the Raman line shape. In our model, the frequency and FWMH of the phonon modes are evaluated from the Raman line shape obtained from Eq. (7).

\section{Density functional calculations}

The phonon dispersion and the PDOS are key elements in the analysis of phonon anharmonic decay. These can, in principle, be calculated to high-accuracy using DFT. Although the proper account of the weak nonlocal van der Waals forces is beyond the usual DFT formulations that rely on the local density approximation (LDA) or the generalized gradient approximation (GGA) [33], LDA has been previously used to study the lattice dynamics of $h$-BN [12,31,34], and it fortuitously yields a very good agreement with the experimental lattice constants [26]. In contrast, the GGA gives essentially no bounding between the hexagonal layers $[33,34]$.

We have performed DFT calculations in the LDA approximation using the ABINIT code [35]. Troulliers-Martins pseudopotentials in the Teter-Pade parametrization were employed. A $9 \times 9 \times 4$ Monkhorst-Pack grid was used for integration over the Brillouin zone, with an energy cutoff of 70 Hartree. For this cut-off energy, the total energy was converged to better than $2 \times 10^{-5}$ Hartree. A full structural relaxation of the $h$-BN lattice yields $a=2.496 \AA$ and $c / a=2.60$. For the calculation of phonon frequencies, the dynamical matrix was obtained on a mesh of $k$ points using the perturbation-theory linear-response approach [36], which allows the interpolation to arbitrary wave vectors for the calculation of phonon dispersion and PDOS.

The $h$-BN crystal belongs to the $P 6_{3} / m m c$ space group and contains four atoms in the unit cell. The optical phonons at the Brillouin zone center can be classified according to the $E_{1 u}+A_{2 u}+2 E_{2 g}+2 B_{1 g}$ irreducible representations. The $E_{1 u}$ and $A_{2 u}$ modes are infrared active whereas the $E_{2 g}$ modes are Raman active and the $B_{1 g}$ modes are silent. The $E_{1 u}$ and $E_{2 g}$ modes are in-plane polarized whereas the $B_{1 g}$ and the $A_{2 u}$ are polarized along the $c$ axis. The calculated phonon dispersion along high-symmetry directions as well as the PDOS are displayed in Fig. 2(a), and the two-phonon DOS for sums $\left(\rho^{+}\right)$and differences $\left(\rho^{-}\right)$of phonons with opposite wave vectors are shown in Fig. 2(b). Along the in-plane $\Sigma$ and $T$ lines, the $E_{2 g}$ modes split into LO and TO branches (labeled $\mathrm{LO}_{1}$ and $\mathrm{TO}_{1}$ for the low-energy mode and $\mathrm{LO}_{2}$ and $\mathrm{TO}_{2}$ for the high-energy mode). Similarly, the polar $E_{1 u}$ mode gives rise to the $\mathrm{TO}_{3}$ and $\mathrm{LO}_{3}$ branches, with a sizable LO-TO splitting at zone center. The $c$-axis polarized $B_{1 g}$ modes give rise to the optical branches labeled as $\mathrm{ZO}_{1}$ and $\mathrm{ZO}_{3}$, corresponding to the low-energy and high-energy modes, respectively, and the $A_{2 u}$ mode gives rise to the $\mathrm{ZO}_{2}$ branch. It should be noted that in Refs. [12,16] the optical branch corresponding to the $B_{1 g}^{\text {low }}$ mode was incorrectly labeled as ZA, and this may lead to confusion in the assignment of spectral features. For instance, in Ref. [16], a second-order Raman feature at $620 \mathrm{~cm}^{-1}$ is assigned to an overtone of an acoustic mode (ZA) at zone center. In Fig. 2, we introduce a systematic labeling scheme for the phonon branches along the $\Sigma$ and $T$ lines to unambiguously identify the phonons that participate in the decay channels discussed in the text. Note that the TA and $\mathrm{TO}_{1}$ branches, $\mathrm{LA}$ and $\mathrm{LO}_{1}$ branches, and $\mathrm{TO}_{2}$ and $\mathrm{TO}_{3}$ branches are nondegenerate. The branches in each pair derive from different zone center modes but they become very close in frequency. For instance, the separation at the midpoint of the $\Sigma$ line for the above pairs of branches is, respectively, 4.3, 0.5 , and $0.3 \mathrm{~cm}^{-1}$. This makes it difficult to clearly distinguish visually between them in the phonon dispersion plot.

The calculations yield zone-center frequencies of 1377 and $50.8 \mathrm{~cm}^{-1}$ for the Raman-active $E_{2 g}^{\text {high }}$ and $E_{2 g}^{\text {low }}$ modes, respectively, in good agreement with the experimental values

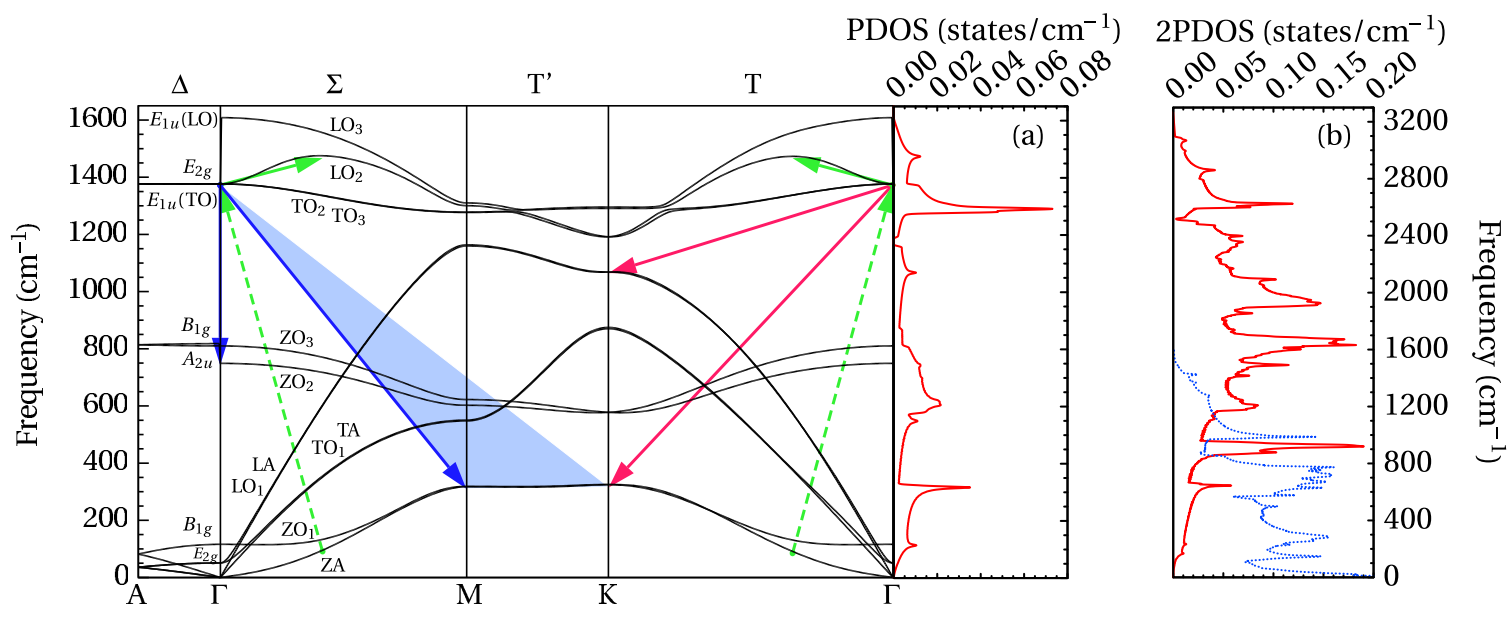

FIG. 2. (a) Phonon dispersion and phonon density of states in $h$-BN as obtained from DFT calculations. The apparent degeneracy of the TA-TO ${ }_{1}, \mathrm{LA}-\mathrm{LO}_{1}$, and $\mathrm{TO}_{2}-\mathrm{TO}_{3}$ branches is discussed in the text. The arrows illustrate the main decay channels for the zone-center $E_{2 g}^{\text {high }}$ mode discussed in the text. Blue arrows indicate the dominant quartic decay process considered. The shaded area depicts the range of possible decay product phonons along the $M-K$ line. The only energetically possible third-order decay process is shown by magenta arrows. Green lines represent a possible upconversion process via annihilation of a ZA phonon. (b) Two-phonon sum (red solid line) and difference (blue dotted line) density of states. 
of 1369 and $52.7 \mathrm{~cm}^{-1}$ measured at low temperature (see Sec. IV A). As expected, the LDA tends to overbind and hence it overestimates the frequency of the $E_{2 g}^{\text {high }}$ mode. On the other hand, the $E_{2 g}^{\text {low }}$ frequency is remarkably well predicted, considering the limitations of LDA to properly describe the interlayer interactions. As a further test to check the applicability of the present LDA calculations to model the lattice dynamics in $h$-BN, we have calculated the bulk modulus. We find $B=-V_{0}(\partial P / \partial V)=26.6 \mathrm{GPa}$, in excellent agreement with the value of 25.6 determined by inelastic $x$-ray scattering [37].

From the inspection of the phonon dispersion, for the $E_{2 g}^{\text {high }}(\Gamma)$ phonon at $1377 \mathrm{~cm}^{-1}$, the only decay channel into two lower-energy phonons that satisfies energy conservation is

$$
E_{2 g}^{\mathrm{high}}(\Gamma) \rightarrow\left\{\begin{array}{c}
\mathrm{LA}^{\mathrm{LO}_{1}} \\
\}
\end{array}(K)+\left\{\begin{array}{c}
\mathrm{ZO}_{1} \\
\mathrm{ZA}
\end{array}\right\}(K)\right.
$$

This decay channel, which is depicted by the magenta arrows in Fig. 2, roughly corresponds to a sharp peak in the sum 2PDOS. In the difference 2 PDOS a peak can also be observed at $\sim 1400 \mathrm{~cm}^{-1}$, which is associated with the three-phonon processes

$$
E_{2 g}^{\text {high }}(\Gamma) \rightarrow \operatorname{LO}_{2}\left\{\begin{array}{c}
(\Gamma-M) \\
(\Gamma-K)
\end{array}\right\}-\mathrm{ZA}\left\{\begin{array}{c}
(\Gamma-M) \\
(\Gamma-K)
\end{array}\right\},
$$

depicted by the green arrows in Fig. 2. In addition, there is a four-phonon decay process that is expected to be relevant on account of the high density of phonon states involved, associated with a flat dispersion of the $\mathrm{ZA}$ and $\mathrm{ZO}_{1}$ branches along the $M-K$ direction. It consists of the decay into a zonecenter $A_{2 u}$ mode and a pair of opposite wave-vector transverseacoustic phonons along the $M-K$ line:

$$
E_{2 g}^{\mathrm{high}}(\Gamma) \rightarrow A_{2 u}(\Gamma)+\left\{\begin{array}{c}
\mathrm{ZA} \\
\mathrm{ZO}_{1}
\end{array}\right\}(M-K)+\left\{\begin{array}{c}
\mathrm{ZA} \\
\mathrm{ZO}_{1}
\end{array}\right\}(M-K) .
$$

This process is indicated by the blue arrows and the shaded region in Fig. 2.

Regarding the $E_{2 g}^{\text {low }}$ modes at $50.8 \mathrm{~cm}^{-1}$, only two up-conversion processes are available for phonon decay: $E_{2 g}^{\text {low }}(\Gamma) \rightarrow B_{1 g}^{\text {high }}(\Gamma)-A_{2 u}(\Gamma)$ and $E_{2 g}^{\text {low }}(\Gamma) \rightarrow \mathrm{ZO}_{2}(M)-$ $\left\{\mathrm{TA} / \mathrm{TO}_{1}\right\}(M)$. Both channels are expected to yield only a minor anharmonic decay contribution to the $E_{2 g}^{\text {low }}$ mode because of the relatively high energies, and thus low occupations, of the phonons that participate in the process. We will consider only the latter, since it entails phonon modes with higher occupation factors and higher density of states.

\section{RESULTS AND DISCUSSION}

\section{A. Raman spectra of the $\boldsymbol{E}_{2 g}$ modes}

The Raman active modes of $h$-BN involve in-plane atomic displacements in the hexagonal layers. As schematically depicted in the inset of Fig. 3, the in-plane vibrations of $\mathrm{B}$ and $\mathrm{N}$ atoms are in antiphase in the $E_{2 g}^{\text {high }}$ mode. Figure 3 displays a set of Raman spectra of the $E_{2 g}^{\text {high }}$ mode obtained at different temperatures between 80 and $600 \mathrm{~K}$. The $E_{2 g}^{\text {high }}$

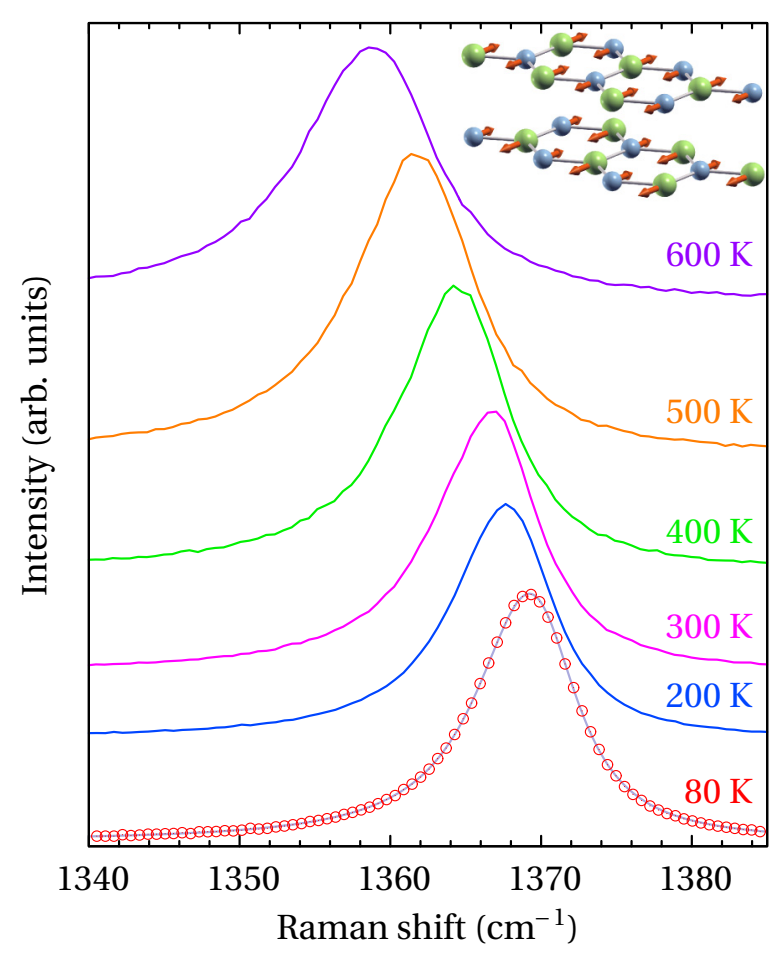

FIG. 3. Raman spectra of the $E_{2 g}^{\text {high }}$ mode obtained at different temperatures in backscattering from a $c$ face. Inset, schematic representation of atomic motion for the $E_{2 g}^{\text {high }}$ mode.

Raman peak shows a progressive downshift and broadening as the temperature is increased. The Raman lineshape is slightly asymmetric with a low-frequency tail. This asymmetry may be due to disorder-induced relaxation of wave-vector conservation or to the frequency dependence of the anharmonic phonon self-energy [19]. The Raman peak parameters [frequency and intrinsic FWHM] are extracted from the fit to the spectra of the convolution of an asymmetric Lorentzian line shape with the spectrometer band-pass broadening of $2.2 \mathrm{~cm}^{-1}$. In Fig. 3, the quality of the fit (gray solid line) is shown on the spectrum recorded at $80 \mathrm{~K}$ (empty circles).

The other Raman active mode of $h$-BN ( $\left.E_{2 g}^{\text {low }}\right)$ corresponds to a gliding motion of the weakly bound hexagonal layers (see inset of Fig. 4), and it exhibits a very low frequency. Figure 4 shows the spectra of the $E_{2 g}^{\text {low }}$ mode recorded at several temperatures. The spectral line shape is well described by a Gaussian function with $\sigma \sim 0.92 \mathrm{~cm}^{-1}$ (solid lines), which corresponds to the spectral bandwidth of the spectrometer. No significant variations of this line shape can be observed in the measurements at different temperatures, and therefore we estimate that in all cases the intrinsic FWHM of this mode is well below the spectrometer bandwidth and does not change appreciably with temperature. This suggests very weak anharmonic interactions for this mode, as is expected from the low probability of the upconversion channels available for phonon decay identified above. However, this mode exhibits a significant frequency downshift as the temperature is increased from 80 to $600 \mathrm{~K}$, which must therefore be due to the thermal lattice expansion effects. Note that the Gaussian fit to the spectra permits the peak frequency to be obtained with a precision significantly higher than the pixel separation in 


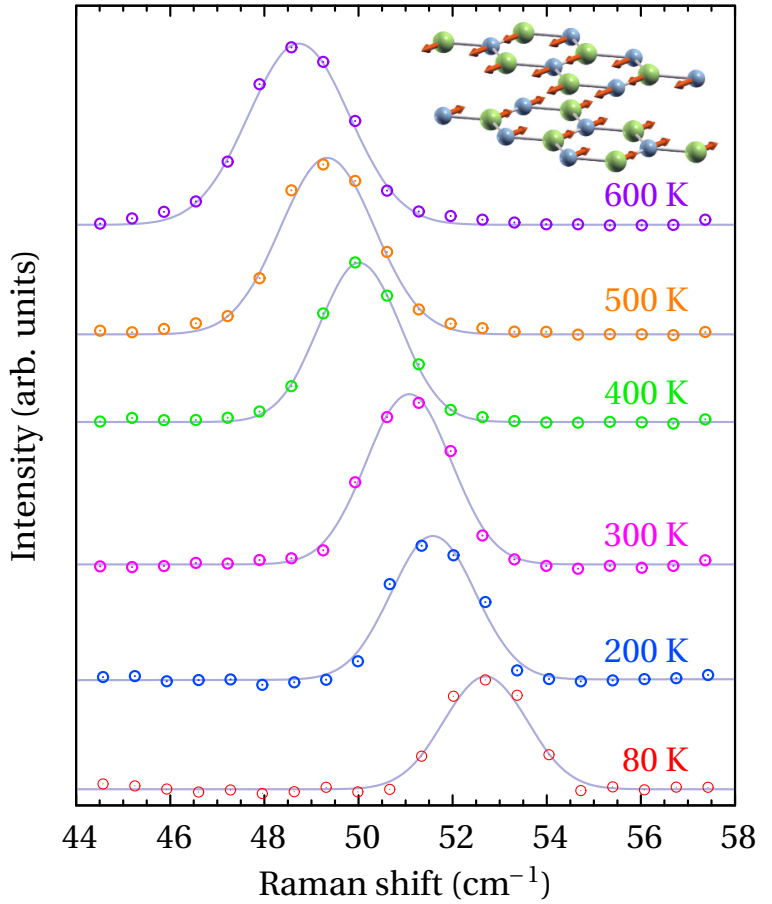

FIG. 4. Raman spectra (empty circles) of the $E_{2 g}^{\text {low }}$ mode obtained at different temperatures in backscattering from a $c$ face. The solid lines correspond to Gaussian line-shape fits to the spectra. Inset, schematic representation of atomic motion for the $E_{2 g}^{\text {low }}$ mode.

the experimental spectra, which allows us to determine the temperature dependence of the frequency with high accuracy.

\section{B. Thermal expansion effects}

As discussed in Sec. III A, layered crystals typically present highly anisotropic thermal expansion behaviors. The thermal expansion coefficient of $h$-BN in the basal plane is negative whereas that in the $c$ direction is positive and one order of magnitude higher than the former [25]. To evaluate the effect of the anisotropic lattice expansion on the frequency of the $E_{2 g}$ modes, we have performed DFT calculations of the phonon frequencies for the lattice parameters corresponding to temperatures between 40 and $620 \mathrm{~K}$. These were obtained from the temperature dependence of the $h$-BN lattice parameter that was experimentally determined in Ref. [25] up to RT by $\mathrm{X}$-ray diffraction using a synchrotron source. For temperatures

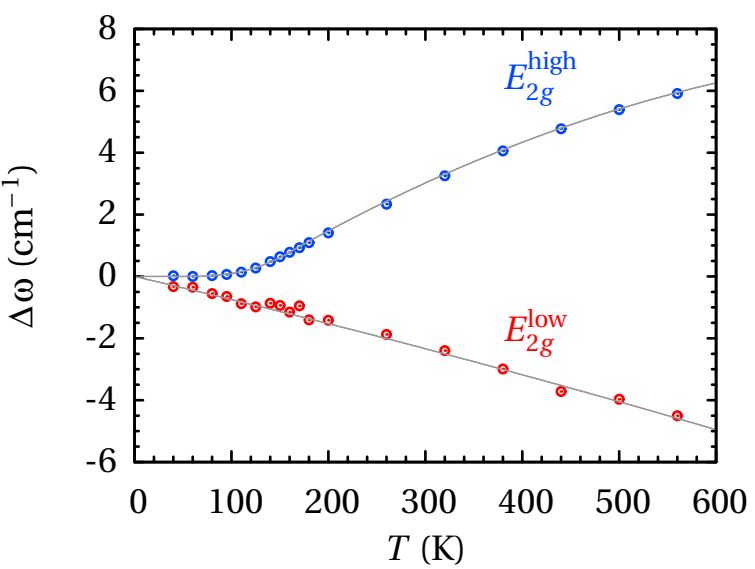

FIG. 5. Calculated frequency shift of the $E_{2 g}$ modes as a function of temperature as obtained by a DFT approach using the experimental lattice parameters determined in Ref. ([25]) and the thermal expansion coefficients reported for temperatures above RT in Ref. ([28]).

from RT up to $600 \mathrm{~K}$, the lattice parameter variation was evaluated using the thermal expansion coefficients reported in the interferometric study by Yates et al. [28]. The temperature dependence of the thermal frequency shift of the $E_{2 g}^{\text {low }}$ phonon calculated by DFT (empty circles in Fig. 5) can be described by fitting a second-order polynomial (solid line in Fig. 5):

$$
\Delta \omega_{l}(T)=-7.317 \times 10^{-3} T-1.565 \times 10^{-6} T^{2} .
$$

The $E_{2 g}^{\text {low }}$ mode, which involves the rigid gliding motion of the hexagonal planes (see inset of Fig. 4), shows a frequency decrease with temperature that reflects the increase of interlayer spacing due to thermal expansion. It is worth noticing that the thermal expansion of the lattice can fully account for the observed shift of the $E_{2 g}^{\text {low }}$ mode. This indicates that anharmonic interactions are very weak for this mode, consistent with the absence of efficient decay channels for such a low-frequency mode.

In contrast, the $E_{2 g}^{\text {high }}$ mode, which entails in-plane antiphase vibrations of boron and nitrogen atoms (see inset of Fig. 3), is primarily affected by the thermal contraction of the in-plane lattice constant, and a substantial frequency increase with temperature is predicted by the calculations. The thermal frequency shift calculated for the $E_{2 g}^{\text {high }}$ mode is best fitted by the following expressions:

$$
\Delta \omega_{h}(T)= \begin{cases}23.474 /[\exp (551.753 / T)-1] & (T \leqslant 160 \mathrm{~K}), \\ -2.347+2.137 \times 10^{-2} T-1.177 \times 10^{-5} T^{2} & (T \geqslant 160 \mathrm{~K}) .\end{cases}
$$

This temperature dependence is in stark contrast with the frequency downshift of the $E_{2}^{\text {high }}$ mode observed in the Raman spectra (see Fig. 3) with increasing temperature. This apparent contradiction can be explained in terms of the strong phonon anharmonic interactions that will be analyzed in the next section.

\section{Anharmonic interactions}

\section{Line broadening and phonon decay channels}

The FWHM of the Raman line shape is inversely proportional to the phonon lifetime and the analysis of its temperature dependence yields information about the phonon anharmonic 


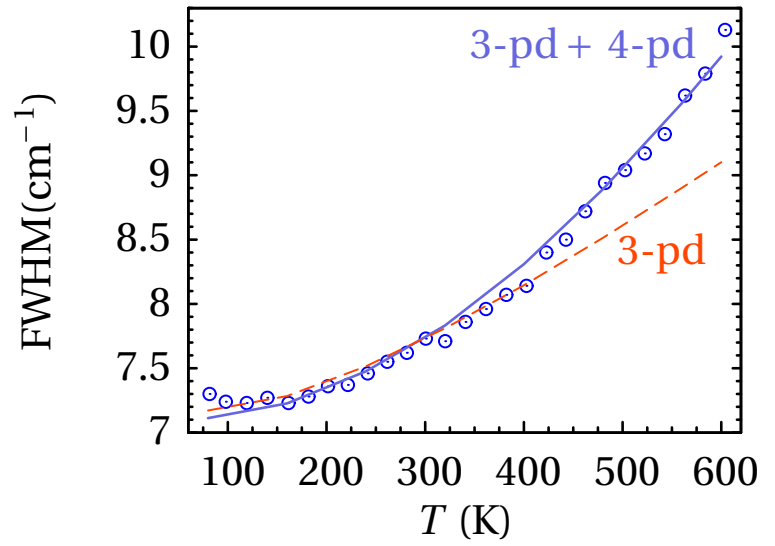

FIG. 6. Temperature dependence of the intrinsic FWHM of the $E_{2 g}^{\text {high }}$ mode obtained from the Raman spectra (empty circles). The dashed line is the temperature dependence predicted by the anharmonic model considering only three-phonon decay processes. The solid line is the fit of the anharmonic model taking into account also four-phonon decay processes.

interactions. In Sec. III B, several possible phonon decay channels were identified on the basis of DFT phonon dispersion calculations. Figure 6 shows the intrinsic FWHM of the $E_{2 g}^{\text {high }}$ mode as a function of temperature determined from the Raman measurements. The rate of FWHM increase with temperature is similar to that reported for a polycrystalline pyrolytic coating [23], but the values of FWHM are consistently $\sim 1.5 \mathrm{~cm}^{-1}$ below those of Ref. [23], which can be ascribed to the higher crystalline quality of our monocrystalline sample. The FWHM of the $E_{2 g}^{\text {high }}$ mode displays a superlinear increase that is notably visible at temperatures above $400 \mathrm{~K}$. Such behavior cannot be explained by the three-phonon decay process discussed in Sec. III C [Eqs. (8) and (9)]. In fact, a least squares fitting of a linear combination of the occupation factors appearing in Eqs. (3) and (4) to the temperature dependence of the FWHM reveals an overwhelming contribution of the four-phonon processes. Thus we consider the quartic anharmonicity decay process specified by Eq. (10) as the dominating decay channel for the $E_{2 g}^{\text {high }}$ mode. A good fit to the FWHM data is obtained with the first term of Eq. (4) for an empirical anharmonic potential of $\left|\tilde{V}_{4}^{+}\right|^{2}=14.5 \mathrm{~cm}^{-2}$ and an additional impurity background broadening of $6.1 \mathrm{~cm}^{-1}$. The values we obtain for the anharmonic coupling potentials are similar to those reported in $c-\mathrm{GaN}$ (Ref. [20]) and in $\mathrm{ZnO}$ [19]. The model fit to the data is plotted as a solid line in Fig. 6. For comparison, the FWHM temperature dependence obtained from the three-phonon processes is also shown as a dashed line.

The FWHM is related to the phonon lifetime through the energy-time uncertainty relation. Phonon lifetime is limited by two main mechanisms, each one with its own characteristic decay time: (i) anharmonic decay into other phonons $\left(\tau_{A}\right)$, and (ii) perturbation of the translational symmetry of the crystal associated with impurities and defects $\left(\tau_{I}\right)$. The FWHM measured in the Raman experiments is then

$$
\mathrm{FWHM}=\frac{\hbar}{\tau}=\hbar\left(\frac{1}{\tau_{A}}+\frac{1}{\tau_{I}}\right),
$$

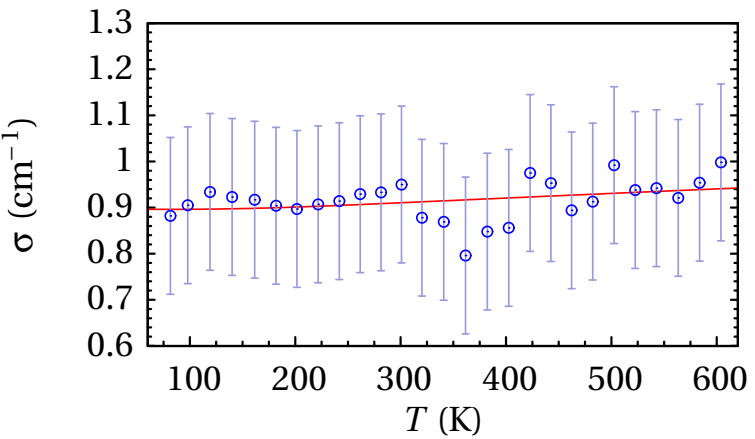

FIG. 7. Temperature dependence of the root-mean-squared width of the Gaussian line shape fitted to the $E_{2 g}^{\text {low }}$ Raman peak (empty circles). The solid line is the fit of the anharmonic model taking into account the upconversion process.

where $\hbar=5.30885 \times 10^{-12} \mathrm{~cm}^{-1} \mathrm{~s}$ is Planck's constant. The impurity relaxation time can be estimated from the impurity background broadening obtained from the above analysis, and we find $\tau_{I} \sim 8.7 \times 10^{-13} \mathrm{~s}$. Then, from the Raman FWHM, the anharmonic relaxation times at RT and $80 \mathrm{~K}$ are determined to be 3.3 and $4.4 \mathrm{ps}$, respectively. These values, which are only slightly higher than those reported for the LO mode of c-GaN (2.0 and $4.2 \mathrm{ps)} \mathrm{[20],} \mathrm{indicate} \mathrm{a} \mathrm{faster} \mathrm{thermalization}$ compared to graphite, where a value of 7 ps was estimated from time-resolved spectroscopy measurements [24].

The FWHM of the $E_{2 g}^{\text {low }}$ peak in the Raman spectra show little variation with temperature. Figure 7 displays the root-mean-squared widths of the Gaussian line shapes that were fitted to the Raman spectra. Error bars have been set to $\pm 0.18 \mathrm{~cm}^{-1}$ to reflect the fact that the experimental points of the spectra deviate in frequency less than one quarter of a pixel in relation to the fitted Gaussian. In an attempt to make a rough estimation of the weak anharmonicity of the $E_{2 g}^{\text {low }}$ mode, and in accordance with the upconversion channel discussed in Sec. III C, a function of the type $\sigma(T)=$ $A+B\left[n_{2}(T)-n_{1}(T)\right]$ was fitted to the data, where $n_{1}(T)$ and $n_{2}(T)$ are, respectively, the Bose-Einstein occupation factors for the $\mathrm{ZO}_{2}$ and TA/TO${ }_{1}$ branches at $M$. This fit suggests a slight increasing trend of the linewidth data points. The relation between the intrinsic FWHM and the $\sigma$ values was obtained from line shape simulations for different FWMH values. From this relation, an upper bound of $\sim 0.11 \mathrm{~cm}^{-1}$ is estimated for the FWHM increase between 80 and $600 \mathrm{~K}$. According to Eq. (3), such broadening would be given by an anharmonicity potential of $\left|V_{3}^{-}\right|^{2}=5.4 \mathrm{~cm}^{-2}$. This is a reasonable value for a third-order difference anharmonic potential, similar to values reported for phonon-difference decay processes in the $E_{2}^{\text {high }}$ mode of $\mathrm{ZnO}$ [19]. The fact that it is significantly lower than the anharmonic potential determined above for the $E_{2 g}^{\text {high }}$ mode confirms that weak anharmonic interactions take place for the $E_{2 g}^{\text {low }}$ mode.

\section{Frequency shift and the role of the four-phonon scattering loop}

Although our previous analysis indicates that anharmonic interactions for the $E_{2 g}^{\text {low }}$ mode are very weak, this mode exhibits a sizable frequency downshift when temperature is increased. As discussed in Sec. IV B, the observed frequency 


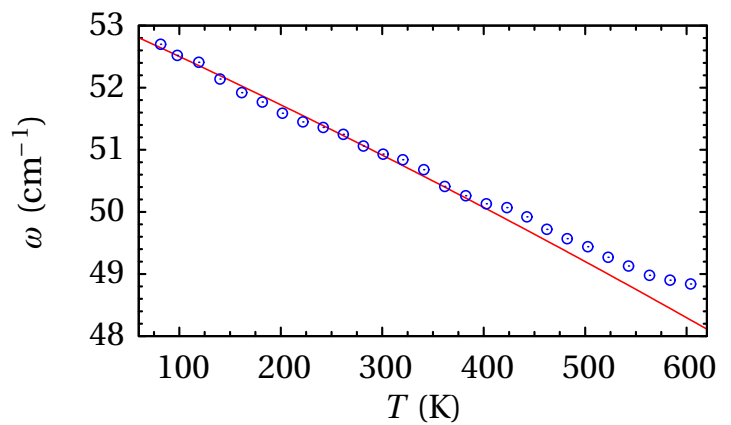

FIG. 8. Temperature dependence of the $E_{2 g}^{\text {low }}$ Raman peak frequency (empty circles). The solid line is the temperature dependence calculated from the thermal expansion of the interlayer distance.

downshift can be fully accounted for by the thermal expansion of the interlayer distance. In Fig. 8, we compare the $E_{2 g}^{\text {low }}$ frequencies obtained from the Raman spectra with the frequency downshift calculated from the thermal expansion of the lattice. The measured $E_{2 g}^{\text {low }}$ frequencies follow closely the temperature behavior expected from the thermal lattice expansion. The slight deviation observed at higher temperatures may be due to the neglected contribution of the first-order self-energy correction [Eq. (6)].

For the $E_{2 g}^{\text {high }}$ mode, in spite of the frequency upshift expected from the in-plane lattice constant decrease with temperature, a sizable frequency downshift is measured in the Raman experiments. The observed rate of frequency decrease with increasing temperature is similar to that reported for a polycrystalline coating [23], but we find frequency values $\sim 4 \mathrm{~cm}^{-1}$ higher than those given in Ref. [23]. Since the $E_{2 g}^{\text {high }}$ frequency that we measure at RT is in good agreement with previously reported values $[14,15]$, we attribute the differences with Ref. [23] to the presence of residual strain and/or impurities in the polycrystalline pyrolytic coating. In Fig. 9, the experimental frequencies of the $E_{2 g}^{\text {high }}$ Raman peaks (empty circles) are compared with the frequency shifts calculated from the lattice thermal expansion (dashed line). From Fig. 9, it is clear that strong anharmonic interactions must affect the $E_{2 g}^{\text {high }}$ mode to reverse the temperature-dependence trend imposed by the thermal expansion of the lattice. However, the Kramers-Kronig transformation [Eq. (5)] of the imaginary self-energy obtained with the anharmonic potentials determined in Sec. IV C 1 yields a negative frequency shift that in absolute value is lower than the thermal contraction shift. The contribution of three- and four-phonon decay processes, which are plotted in Fig. 9 with double-dashed and dot-dashed lines, respectively, are clearly insufficient to reverse the lattice expansion trend. Therefore the origin of the large negative frequency shift observed in the Raman measurements must lie in the purely real contribution to the phonon self-energy arising from the first-order, four-phonon scattering term [Eq. (6)]. A similar situation has been reported in graphite [21], in which both the lattice expansion and the three-phonon decay contributions to the frequency shift are positive for temperatures up to $600 \mathrm{~K}$, but the strong negative shift due to the four-phonon scattering dominates and results

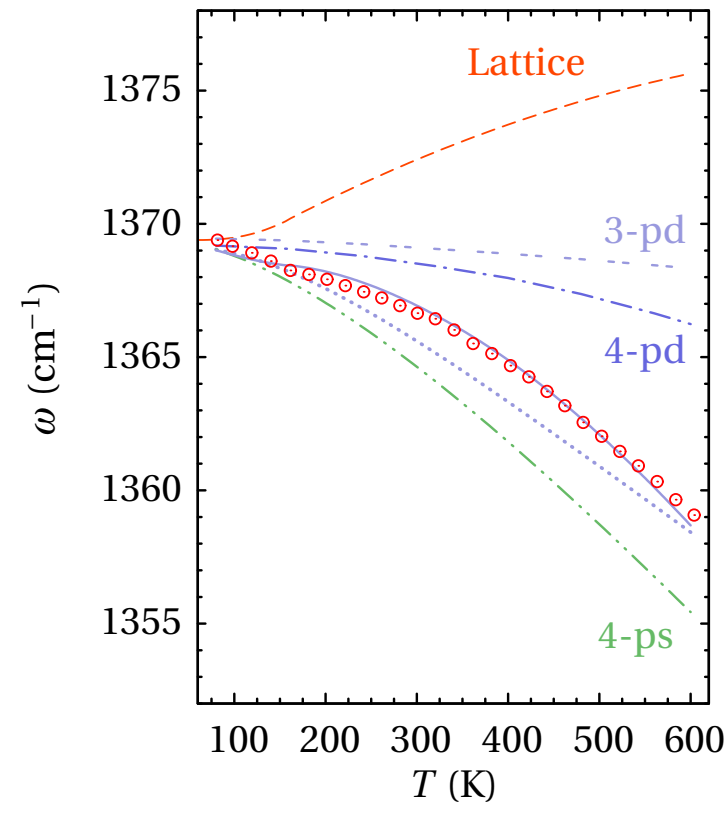

FIG. 9. Temperature dependence of the $E_{2 g}^{\text {high }}$ Raman peak frequency (empty circles). The solid line is the full model fit to the data. The dashed line indicates the frequency shift associated with thermal lattice expansion. The double-dashed and dot-dashed lines represent, respectively, the temperature dependence of the contribution of three-phonon (3-pd) and four-phonon (4-pd) decay processes. The dashed-dot-dotted line is the first-order four-phonon scattering (4-ps) contribution fitted to the data. The dotted line is an $a b$ initio estimate of the latter contribution.

in a marked frequency downshift of the $E_{2 g}^{\text {high }}$ mode with the temperature.

To substantiate the explanation of the $E_{2 g}^{\text {high }}$ temperature downshift in $h$-BN as due to a strong four-phonon scattering anharmonic interaction, the corresponding contribution to the real phonon self-energy [Eq. (6)] has been estimated using the results of our DFT calculations. The matrix elements $V^{(4)}\left(\mathbf{0} j_{0}, \mathbf{0} j_{0}, \boldsymbol{q} j,-\boldsymbol{q} j\right)$ in Eq. (6) are related to the fourth derivatives of the interatomic potential with respect to the atomic displacements associated with the phonon modes [17]. A full evaluation of Eq. (6) is beyond the scope of the present work. Nevertheless, assuming dispersionless anharmonic potentials, an estimation of the four-phonon scattering contribution to the frequency shift of the $i$ th phonon mode can be obtained as

$$
\Delta_{i}^{4 \mathrm{ps}} \approx \frac{1}{2 \hbar} \sum_{j} \int_{\omega_{j}}^{\omega_{j+1}} d \omega \frac{\partial^{4} E}{\partial u_{i}^{2} \partial u_{j}^{2}}[2 n(\omega)+1] \rho(\omega)
$$

where the sum runs over all phonon branches, $E$ is the interatomic potential energy, and the adimensional phonon displacements $u_{j}$ are defined by

$$
u_{j}=\sum_{s, \alpha} \sqrt{\frac{2 M_{s} \omega_{j}}{\hbar}} e_{s \alpha}^{j} .
$$

Here, the fourth derivatives are evaluated at zone center and $e_{s \alpha}^{j}$ is the eigenvector of the $j$ th phonon mode normalized to the 


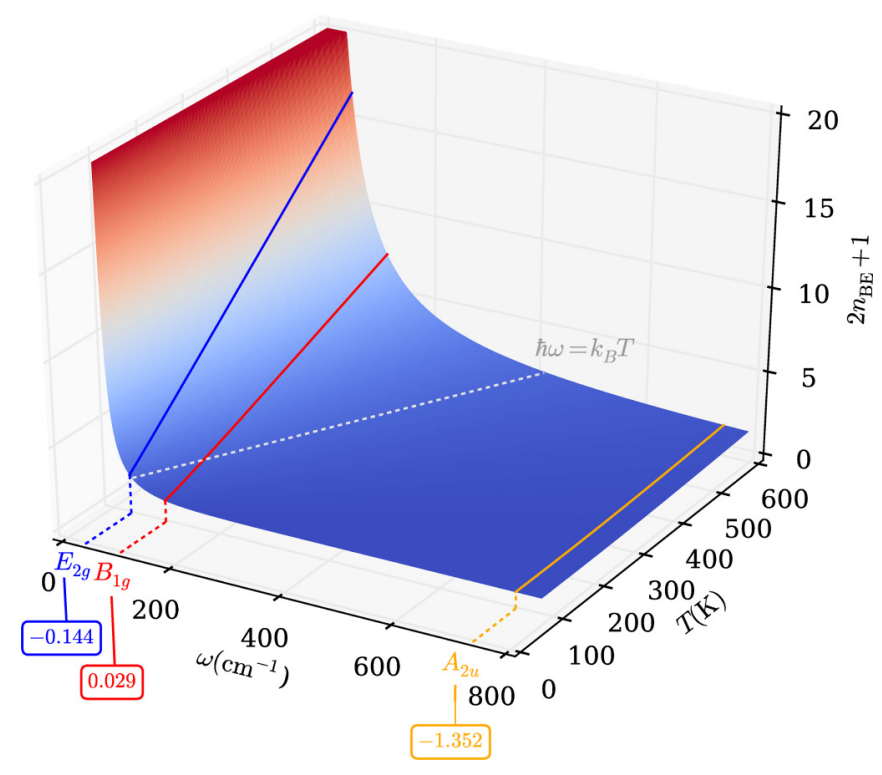

FIG. 10. Plot of the occupation factor $2 n+1$ as a function of temperature and phonon energy. The frequencies of the $E_{2 g}^{\text {low }}, B_{1 g}^{\text {low }}$, and $A_{2 u}$ modes are indicated. The numbers in the boxes are the respective coupling potentials (expressed in Hartree units) of these modes with the $E_{2 g}^{\text {high }}$ mode for the four-phonon scattering mechanism.

unit cell. The indices $s$ and $\alpha$ denote, respectively, the atomic site and the cartesian coordinates within the unit cell.

The temperature dependence in Eq. (14) arises from the Bose-Einstein occupation factor, which is only appreciable for low phonon energies, $\hbar \omega(\boldsymbol{q} j) \lesssim k_{B} T$. Therefore the temperature shift of the four-phonon scattering term is primarily governed by coupling to the lower lying modes, since the occupation of these modes changes more strongly in the temperature range studied. This is illustrated in Fig. 10, where the occupation factor $2 n+1$ is plotted as a function of temperature and phonon frequency. As can be seen in Fig. 10, the occupation factor $2 n+1$ is significantly larger than 1 only for $T \gtrsim \hbar \omega / k_{\mathrm{B}}$. Consequently, only for the $E_{2 g}^{\text {low }}$ and $B_{1 g}^{\text {low }}$ modes the occupation factors display a substantial variation over the temperature range studied. As can be seen from Eq. (14), the sign of the coupling potential $\left(\partial^{4} E / \partial u_{i}^{2} \partial u_{j}^{2}\right)$ to the lowest lying modes will therefore determine the sign of the frequency correction associated with the four-phonon scattering loop.

Layered compounds like graphite or $h$-BN exhibit characteristic low-frequency optical branches associated with the existence of modes involving the rigid motion of the hexagonal layers, which are ruled by the weak interlayer interactions. Thus it is expected that scattering with the low-lying optical modes will be important in these type of crystals. Indeed, detailed DFT calculations based on the full evaluation of Eq. (6) have shown that the temperature downshift of the $E_{2 g}$ frequency in graphite is almost entirely determined by the four-phonon scattering term [21].

To gain insight into the relevance of this term in $h$-BN, we first perform a simple semi-empirical fit of the loop correction [Eq. (6)] to the experimental data. By considering that the four-phonon scattering is dominated by the lower lying modes, and further assuming that the anharmonic coupling does not differ substantially between these modes and thus can be represented by an average effective anharmonic potential, the temperature dependence of the frequency shift (relative to that corresponding to $T_{0}$ ) arising from four-phonon scattering can be approximated by

$$
\Delta_{T_{0}}^{4 \mathrm{ps}}(T) \approx \tilde{V}^{4 \mathrm{ps}} \int_{0}^{\infty} d \omega\left[n(\omega, T)-n\left(\omega, T_{0}\right)\right] \rho(\omega) .
$$

By including the additional contribution $\Delta_{T_{0}}^{4 \mathrm{ps}}(T)$ to the frequency shift resulting from lattice expansion and threeand four-phonon anharmonic decay, a very good fit with the frequency downshift data is obtained for $\tilde{V}^{4 \mathrm{ps}}=-2.21 \mathrm{~cm}^{-1}$. This is shown as a solid line in Fig. 9, while the separate contribution of $\Delta_{T_{0}}^{4 \mathrm{ps}}(T)$ is plotted as a dot-dot-dashed line.

Next, we compare the semi-empirical fit (dot-dot-dashed line in Fig. 9) with an $a b$ initio estimate of the four-phonon scattering term based on Eq. (14) (dotted line in Fig. 9). This can be obtained from Eq. (14) by evaluating the fourth derivatives of the interatomic potential energy using finite differences of the second derivative values calculated by the ABINIT code at the Brillouin zone center. The derivatives with respect to the adimensional phonon coordinates $u_{j}$ are related to the derivatives with respect to the reduced (i.e., scaled by the lattice parameter) atomic coordinates $\xi_{s}$ by

$$
\frac{\partial}{\partial u_{j}}=\sum_{s, \alpha} \sqrt{\frac{\hbar}{2 M_{s} \omega_{j}}} e_{s \alpha}^{j} \frac{1}{d_{\alpha}} \frac{\partial}{\partial \xi_{s \alpha}},
$$

where $d_{\alpha}$ equals $a$ or $c$ depending on the polarization direction of the phonon mode. For simplicity, we align the polarization of the doubly degenerate in-plane modes along the Cartesian $x$ axis. Using a frozen-phonon approach, the second derivatives are calculated at atomic positions displaced by a small fraction $\left(\delta= \pm 1 \times 10^{-3}\right)$ of the reduced phonon eigenvectors. Then, an estimation of the fourth-order derivatives with respect to the Cartesian coordinates is obtained by finite differences, and suitable linear combinations of these according to Eq. (17) yield the fourth-order derivatives with respect the zone-center phonon displacements that enter in Eq. (14).

The values of the fourth-order derivatives we obtain for the lower lying modes are displayed in the boxes in Fig. 10 (expressed in Hartree units). For the $E_{2 g}^{\text {high }}$ mode, a negative anharmonic coupling potential with the lowest-lying $E_{2 g}^{\text {low }}$ mode is found, whereas the coupling is positive but very small for the $B_{1 g}^{\text {low }}$ mode. As already discussed above, the sign of the coupling potential determines the sign of the frequency shift associated with the four-phonon scattering loop. Consequently, the temperature dependence is dominated in this case by the negative coupling to the $E_{2 g}^{\text {low }}$ mode, and a frequency downshift with increasing temperature results from the four-phonon anharmonic correction. The frequency shift evaluated using Eq. (14) is plotted as a dotted line in Fig. 9. Considering the approximations made in the calculations, the ab-initio theoretical estimate is in good agreement with the empirical fit to the Raman data (dot-dot-dashed line). This confirms that the anharmonic coupling to the lower lying modes through four-phonon scattering governs the temperature-induced shift observed in the $E_{2 g}^{\text {high }}$ mode. 


\section{SUMMARY AND CONCLUSIONS}

We have performed a study of anharmonic interactions in the layered $h$-BN crystal by means of temperature-dependent Raman scattering measurements in the range between 80 and $600 \mathrm{~K}$. The results are analyzed using Cowley's perturbation theory. The most likely decay channels are identified from DFT phonon dispersion calculations. The highly anisotropic thermal expansion of the $h$-BN crystal is taken into account.

Both $E_{2 g}$ Raman active modes of $h$-BN are detected and analyzed. The interlayer low-frequency mode $E_{2 g}^{\text {low displays }}$ very narrow linewidth and weak anharmonic interactions. Its frequency downshift with temperature is fully accounted for by the increase in interlayer spacing, and it shows an insignificant broadening in the temperature range studied. This behavior is consistent with the lack of efficient phonon decay channels for such a low-energy mode. In contrast, the $E_{2 g}^{\text {high }}$ mode exhibits a sizable broadening with temperature that can only be explained by taking into account four-phonon decay processes (quartic anharmonicity). The analysis of the $E_{2 g}^{\text {high }}$ Raman linewidth indicates a faster phonon relaxation compared to graphite.
The strong anharmonic interactions that take place in the $h$-BN layered crystal reverse the effect of the in-plane lattice parameter reduction with increasing temperature and yield a marked frequency downshift of the $E_{2 g}^{\text {high }}$ mode. Similar to the case of the $E_{2 g}$ mode of graphite and graphene, the fourphonon scattering contribution to the quartic anharmonicity plays a major role in determining the temperature dependence of the $E_{2 g}^{\text {high }}$ frequency in $h$-BN. This effect, which is negligible in other wide band-gap nitrides such as, for instance, $c-\mathrm{GaN}$, acquires relevance in layered crystals such as $h$-BN and graphite because of the existence of very low frequency modes associated with weak interlayer interactions. In fact, in $h$-BN the negative coupling potential to the $E_{2 g}^{\text {low }}$ mode dominates the loop term and explains the strong frequency downshift with temperature observed for the $E_{2 g}^{\text {high }}$ mode.

\section{ACKNOWLEDGMENTS}

This work has been financially supported by the Spanish MINECO under Contract No. MAT2015-71035-R. and by the network GaNeX (ANR-11-LABX-0014). GaNeX belongs to the publicly funded Investissements d'Avenir program managed by the French ANR agency.
[1] G.-H. Lee, Y.-J. Yu, X. Cui, N. Petrone, C.-H. Lee, M. S. Choi, D.-Y. Lee, C. Lee, W. J. Yoo, K. Watanabe, T. Taniguchi, C. Nuckolls, P. Kim, and J. Hone, ACS Nano 7, 7931 (2013).

[2] O. Hod, J. Chem. Theory Comput. 8, 1360 (2012).

[3] N. Marom, J. Bernstein, J. Garel, A. Tkatchenko, E. Joselevich, L. Kronik, and O. Hod, Phys. Rev. Lett. 105, 046801 (2010).

[4] K. Watanabe, T. Taniguchi, and H. Kanda, Nat. Mater. 3, 404 (2004).

[5] G. Cassabois, P. Valvin, and B. Gil, Nat. Photonics 10, 262 (2016).

[6] G. Cassabois, P. Valvin, and B. Gil, Phys. Rev. B 93, 035207 (2016).

[7] W. Gannett, W. Regan, K. Watanabe, T. Taniguchi, M. F. Crommie, and A. Zettl, Appl. Phys. Lett. 98, 242105 (2011).

[8] T. Roy, M. Tosun, J. S. Kang, A. B. Sachid, S. B. Desai, M. Hettick, C. C. Hu, and A. Javey, ACS Nano 8, 6259 (2014).

[9] C. R. Dean, A. F. Young, I. Meric, C. Lee, L. Wang, S. Sorgenfrei, K. Watanabe, T. Taniguchi, P. Kim, K. L. Shepard, and J. Hone, Nat. Nanotechnol. 5, 722 (2010).

[10] L. Viti, J. Hu, D. Coquillat, A. Politano, C. Consejo, W. Knap, and M. S. Vitiello, Adv. Mater. 28, 7390 (2016).

[11] I. Jo, M. T. Pettes, J. Kim, K. Watanabe, T. Taniguchi, Z. Yao, and L. Shi, Nano Lett. 13, 550 (2013).

[12] J. Serrano, A. Bosak, R. Arenal, M. Krisch, K. Watanabe, T. Taniguchi, H. Kanda, A. Rubio, and L. Wirtz, Phys. Rev. Lett. 98, 095503 (2007).

[13] R. Geick, C. H. Perry, and G. Rupprecht, Phys. Rev. 146, 543 (1966).

[14] T. Kuzuba, K. Era, T. Ishii, and T. Sato, Solid State Commun. 25, 863 (1978).

[15] R. J. Nemanich, S. A. Solin, and R. M. Martin, Phys. Rev. B 23, 6348 (1981).
[16] S. Reich, A. C. Ferrari, R. Arenal, A. Loiseau, I. Bello, and J. Robertson, Phys. Rev. B 71, 205201 (2005).

[17] J. Menéndez and M. Cardona, Phys. Rev. B 29, 2051 (1984).

[18] L. Bergman, D. Alexson, P. L. Murphy, R. J. Nemanich, M. Dutta, M. A. Stroscio, C. Balkas, H. Shin, and R. F. Davis, Phys. Rev. B 59, 12977 (1999).

[19] R. Cuscó, E. Alarcón-Lladó, J. Ibáñez, L. Artús, J. Jiménez, B. Wang, and M. J. Callahan, Phys. Rev. B 75, 165202 (2007).

[20] R. Cuscó, N. Domènech-Amador, S. Novikov, C. T. Foxon, and L. Artús, Phys. Rev. B 92, 075206 (2015).

[21] P. Giura, N. Bonini, G. Creff, J. B. Brubach, P. Roy, and M. Lazzeri, Phys. Rev. B 86, 121404(R) (2012).

[22] S. Linas, Y. Magnin, B. Poinsot, O. Boisron, G. D. Förster, V. Martinez, R. Fulcrand, F. Tournus, V. Dupuis, F. Rabilloud, L. Bardotti, Z. Han, D. Kalita, V. Bouchiat, and F. Calvo, Phys. Rev. B 91, 075426 (2015).

[23] G. J. Exarhos, and J. W. Schaaf, J. Appl. Phys. 69, 2543 (1991).

[24] T. Kampfrath, L. Perfetti, F. Schapper, C. Frischkorn, and M. Wolf, Phys. Rev. Lett. 95, 187403 (2005).

[25] W. Paszkowicz, J. Pelka, M. Knapp, T. Szyszko, and S. Podsiadlo, Appl. Phys. A 75, 431 (2002).

[26] N. Mounet and N. Marzari, Phys. Rev. B 71, 205214 (2005).

[27] W. Bao, F. Miao, Z. Chen, H. Zhang, W. Jang, C. Dames, and C. N. Lau, Nat. Nanotechnol. 4, 562 (2009).

[28] B. Yates, M. J. Overy, and O. Pirgon, Philos. Mag. 32, 847 (1975).

[29] R. A. Cowley, Rep. Prog. Phys. 31, 123 (1968).

[30] N. Domènech-Amador, R. Cuscó, L. Artús, T. Yamaguchi, and Y. Nanishi, Phys. Rev. B 83, 245203 (2011).

[31] N. Bonini, M. Lazzeri, N. Marzari, and F. Mauri, Phys. Rev. Lett. 99, 176802 (2007). 
[32] M. Balkanski, R. F. Wallis, and E. Haro, Phys. Rev. B 28, 1928 (1983).

[33] H. Rydberg, M. Dion, N. Jacobson, E. Schröder, P. Hyldgaard, S. I. Simak, D. C. Langreth, and B. I. Lundqvist, Phys. Rev. Lett. 91, 126402 (2003).

[34] G. Kern, G. Kresse, and J. Hafner, Phys. Rev. B 59, 8551 (1999).

[35] ABINIT is a common project of the Universite Catholique de Louvain, Corning Incorporated, and other contributors (http://www.abinit.org). X. Gonze, J.-M. Beuken, R. Caracas, F. Detraux, M. Fuchs, G.-M. Rignanese, L. Sindic, M. Verstraete, G. Zerah, F. Jollet, M. Torrent, A. Roy, M. Mikami, Ph. Ghosez, J.-Y. Raty, and D. C. Allan, Comput. Mater. Sci. 25, 478 (2002).

[36] X. Gonze and C. Lee, Phys. Rev. B 55, 10355 (1997).

[37] A. Bosak, J. Serrano, M. Krisch, K. Watanabe, T. Taniguchi, and H. Kanda, Phys. Rev. B 73, 041402(R) (2006). 